PROCEEDINGS OF THE

AMERICAN MATHEMATICAL SOCIETY

Volume 134, Number 11, November 2006, Pages 3301-3307

S 0002-9939(06)08355-9

Article electronically published on May 12, 2006

\title{
SOME UPPER BOUNDS FOR SUMS OF EIGENVALUES OF THE NEUMANN LAPLACIAN
}

\author{
LIANGPAN LI AND LAN TANG
}

(Communicated by Richard A. Wentworth)

\begin{abstract}
Let $\mu_{k}(\Omega)$ be the $k$ th Neumann eigenvalue of a bounded domain $\Omega$ with piecewisely smooth boundary in $\mathbf{R}^{n}$. In 1992, P. Kröger proved that $k^{-\frac{n+2}{n}} \sum_{j=1}^{k} \mu_{j} \leq \frac{4 n \pi^{2}}{n+2}\left(\omega_{n} V\right)^{-2 / n}$, where the upper bound is sharp in view of Weyl's asymptotic formula. The aim of this paper is twofold. First, we will improve this estimate by multiplying a factor in terms of $k$ to its right-hand side which approaches strictly from below to 1 as $k$ tends to infinity. Second, we will generalize Kröger's estimate to the case when $\Omega$ is a compact Euclidean submanifold.
\end{abstract}

\section{INTRODUCTION}

Let $\Omega \subset \mathbf{R}^{n}$ be a bounded domain with piecewisely smooth boundary. The Neumann eigenvalue problem

$$
\begin{array}{rll}
\Delta u+\mu u=0 & \text { in } \Omega, \\
\frac{\partial u}{\partial \nu}=0 & \text { on } \partial \Omega
\end{array}
$$

has eigenvalues $0=\mu_{1}<\mu_{2} \leq \mu_{3} \leq \cdots$ (here $\partial / \partial \nu$ denotes the outward normal derivative on $\partial \Omega$ ). Let $V$ and $\omega_{n}$ denote the volume of $\Omega$ and the unit ball in $\mathbf{R}^{n}$, respectively. Then the asymptotic formula of Weyl [10] asserts that the eigenvalues of problem (1.1) satisfy the following formula:

$$
\lim _{k \rightarrow \infty} k^{-2 / n} \mu_{k}=4 \pi^{2}\left(\omega_{n} V\right)^{-2 / n}
$$

From (1.2), it follows at once that

$$
\lim _{k \rightarrow \infty} k^{-\frac{n+2}{n}} \sum_{j=1}^{k} \mu_{j}=\frac{4 n \pi^{2}}{n+2}\left(\omega_{n} V\right)^{-2 / n} .
$$

In [6], Kröger has shown that for problem (1.1) the inequality

$$
k^{-\frac{n+2}{n}} \sum_{j=1}^{k} \mu_{j} \leq \frac{4 n \pi^{2}}{n+2}\left(\omega_{n} V\right)^{-2 / n}
$$

holds for all $k$. This result is consistent with (1.3) and is remarkable in that it shows that the limit in (1.3) is always approached from below.

Received by the editors November 1, 2004 and, in revised form, May 28, 2005.

2000 Mathematics Subject Classification. Primary 35P15; Secondary 58G25.

Key words and phrases. Eigenvalue, Neumann Laplacian.

(C)2006 American Mathematical Society 3301

Reverts to public domain 28 years from publication 
It is the purpose of this paper to extend the estimate (1.4) in two different directions. First, we will obtain an improvement of (1.4) to solve a question raised by Hermi [3] to some extent. Namely,

Theorem 1.1. For any bounded domain $\Omega \subset \mathbf{R}^{n}$ with piecewisely smooth boundary and any $k \geq 1$ one has

$$
k^{-\frac{n+2}{n}} \sum_{j=1}^{k} \mu_{j} \leq \frac{4 n \pi^{2}}{n+2}\left(\omega_{n} V\right)^{-2 / n} C(k, n, \Omega),
$$

where the constant $C(k, n, \Omega)<1$ depends only on $k, n$ and $\Omega$.

Second, let $M^{m}(1 \leq m \leq n)$ be an $m$-dimensional compact, regular submanifold of $\mathbf{R}^{n}$ with smooth boundary $\partial M$ ( $\partial M$ may be empty). It is well known that the intrinsic-defined Laplace operator $\triangle$ under Neumann boundary condition

$$
\begin{array}{rlr}
\Delta u+\widetilde{\mu} u & =0 & \text { in } M^{m}, \\
\frac{\partial u}{\partial \nu} & =0 & \text { on } \partial M
\end{array}
$$

has discrete eigenvalues $0=\widetilde{\mu_{1}}<\widetilde{\mu_{2}} \leq \widetilde{\mu_{3}} \leq \cdots$ (see [9, page 88]). Also denote by $V$ the volume of $M^{m}$. Similar to (1.4), we have

Theorem 1.2. Under the above assumptions, one has

$$
\frac{1}{k} \sum_{j=1}^{k} \widetilde{\mu}_{j} \leq \frac{n}{n+2}\left(\frac{k C}{\omega_{n} V}\right)^{2 / m},
$$

where $C$ depends only on $M^{m}$ and $n$. In particular, $C=(2 \pi)^{n}$ if $m=n$.

Since the problems of obtaining upper bounds for Neumann eigenvalues and the lower bounds for Dirichlet eigenvalues are intertwining with each other, we mention some known results on Dirichlet eigenvalues as a counterpart. Let $0<\lambda_{1}<\lambda_{2} \leq$ $\lambda_{3} \leq \cdots$ denote the eigenvalues of the problem

$$
\begin{array}{rll}
\triangle u+\lambda u=0 & \text { in } \Omega, \\
u=0 & \text { on } \partial \Omega .
\end{array}
$$

We also have

$$
\begin{aligned}
& \lim _{k \rightarrow \infty} k^{-2 / n} \lambda_{k}=4 \pi^{2}\left(\omega_{n} V\right)^{-2 / n}, \\
& k^{-\frac{n+2}{n}} \sum_{j=1}^{k} \lambda_{j} \geq \frac{4 n \pi^{2}}{n+2}\left(\omega_{n} V\right)^{-2 / n}, \quad \forall k,
\end{aligned}
$$

by Weyl [10] and Li and Yau [7, respectively. Recently, Melas [8] improved the estimate (1.10) to the following:

$$
k^{-\frac{n+2}{n}} \sum_{j=1}^{k} \lambda_{j} \geq \frac{4 n \pi^{2}}{n+2}\left(\omega_{n} V\right)^{-2 / n}+B_{n} \frac{V}{I} k^{-\frac{2}{n}}, \forall k,
$$

where the constant $B_{n}$ depends only on the dimension $n$ and $I$ is the "moment of inertia" of $\Omega$, that is, $I=\min _{a \in \mathbf{R}^{n}} \int_{\Omega}|x-a|^{2} d x$. Though estimates (1.5) and (1.11) again show the analogy between the problems mentioned above, we do not know yet how to generalize (1.10) to the case when $M^{m}$ is of dimension $m<n$. 


\section{Proof of Theorem 1.1}

We first introduce a lemma, which is useful to the proofs of both Theorem 1.1 and Theorem 1.2.

Lemma 2.1. Let $\mu_{1} \leq \mu_{2} \leq \cdots \leq \mu_{k+1}$ be some nonnegative numbers such that

$$
\mu_{k+1} \leq \frac{a-\sum_{j=1}^{k} \mu_{j} c_{j}}{b-\sum_{j=1}^{k} c_{j}}
$$

for some positive numbers $a, b, c_{j}$ with $c_{j} \leq c$. Assume moreover that $b>k c$. Then

$$
c \cdot \sum_{j=1}^{k} \mu_{j} \leq a .
$$

Proof of Lemma 2.1. By the assumptions $c_{j} \leq c, b>k c$, we have $b-\sum_{j=1}^{k} c_{j}>0$. Since $\mu_{k+1} \geq 0$, it follows that

$$
\mu_{k+1}\left(b-\sum_{j=1}^{k} c_{j}\right) \leq a-\sum_{j=1}^{k} \mu_{j} c_{j} .
$$

Equivalently,

$$
\mu_{k+1} b-a \leq \sum_{j=1}^{k}\left(\mu_{k+1}-\mu_{j}\right) c_{j} .
$$

Consequently, by the assumptions $\mu_{j} \leq \mu_{k+1}, c_{j} \leq c$, we have

$$
\mu_{k+1} b-a \leq \sum_{j=1}^{k}\left(\mu_{k+1}-\mu_{j}\right) c
$$

which is equivalent to

$$
\mu_{k+1}(b-k c) \leq a-\left(\sum_{j=1}^{k} \mu_{j}\right) c .
$$

Hence Lemma 2.1 follows from the assumptions $\mu_{k+1} \geq 0$ and $b>k c$ immediately.

Now we turn to the proof of Theorem 1.1.

Let $\left\{\phi_{j}\right\}_{j=1}^{k}$ be a set of orthonormal real-valued eigenfunctions corresponding to the eigenvalues $\mu_{1}, \mu_{2}, \ldots, \mu_{k}$. Consider the function defined by

$$
\Phi_{k}(x, y) \equiv \sum_{j=1}^{k} \phi_{j}(x) \phi_{j}(y), \text { for } x, y \in \Omega .
$$

The projection of $h_{z}(y) \equiv e^{i\langle z, y\rangle}$ onto the subspace of $L^{2}(\Omega)$ spanned by $\phi_{1}, \phi_{2}, \ldots$, $\phi_{k}$ can be written in terms of the Fourier transform $\widehat{\Phi_{k}}$ of $\Phi_{k}$ with respect to the $x$-variable:

$$
\int_{\Omega} e^{i\langle z, x\rangle} \Phi_{k}(x, y) d x=\widehat{\Phi_{k}}(z, y)
$$


Quoting the analysis in Kröger [6], one has

$$
\begin{aligned}
\mu_{k+1} & \leq \inf _{r>0} \frac{\int_{B_{r}} \int_{\Omega}\left|\nabla_{y}\left(h_{z}(y)-\widehat{\Phi_{k}}(z, y)\right)\right|^{2} d y d z}{\int_{B_{r}} \int_{\Omega}\left|h_{z}(y)-\widehat{\Phi_{k}}(z, y)\right|^{2} d y d z} \\
& \leq \frac{\frac{n \omega_{n} V}{n+2} r^{n+2}-\sum_{j=1}^{k} \mu_{j} \int\left|\hat{\phi}_{j}\right|^{2}}{\omega_{n} V r^{n}-\sum_{j=1}^{k} \int\left|\hat{\phi}_{j}\right|^{2}}
\end{aligned}
$$

here $B_{r}$ denotes the ball centered at 0 in $\mathbf{R}^{n}$ with radius $r$. Set $r^{*}=r^{*}(k)=$ $2 \pi\left(\frac{k}{\omega_{n} V}\right)^{1 / n}$. By a qualitative theorem of Amrein-Berthier on the uncertainty principle in the third chapter of the first part of Havin and Jöricke [2, there exists a constant $c\left(r^{*}\right)<(2 \pi)^{n}$ depending only on the radius $r^{*}$ and the dimension $n$ such that for all $j \leq k$,

$$
\int_{B_{r^{*}}}\left|\widehat{\phi_{j}}(z)\right|^{2} d z \leq c\left(r^{*}\right)
$$

An estimate of an upper bound for the constant $c\left(r^{*}\right)$ is also given in the last part of this section. Set $\widetilde{r}=\widetilde{r}(k)=\left(\frac{k c\left(r^{*}\right)}{\omega_{n} V}\right)^{1 / n}=\frac{\sqrt[n]{c\left(r^{*}\right)}}{2 \pi} r^{*}$, and always assume $\widetilde{r}<r<r^{*}$ from now. Since $\tilde{r}<r<r^{*}, \omega_{n} V r^{n}-k c\left(r^{*}\right)>0$. By Lemma 2.1,

$$
\frac{n \omega_{n} V}{n+2} r^{n+2}-c\left(r^{*}\right) \sum_{j=1}^{k} \mu_{j} \geq 0,
$$

which yields

$$
\begin{aligned}
\sum_{j=1}^{k} \mu_{j} & \leq \inf _{\tilde{r}<r<r^{*}} \frac{n \omega_{n} V r^{n+2}}{(n+2) c\left(r^{*}\right)} \\
& =\frac{n \omega_{n} V \widetilde{r}^{n+2}}{(n+2) c\left(r^{*}\right)} \\
& =\frac{n}{n+2}\left(\frac{c\left(r^{*}\right)}{\omega_{n} V}\right)^{2 / n} k^{\frac{n+2}{n}} .
\end{aligned}
$$

Corresponding to the inequality (1.4), we have

$$
k^{-\frac{n+2}{n}} \sum_{j=1}^{k} \mu_{j} \leq \frac{n}{n+2}\left(\frac{c\left(r^{*}\right)}{\omega_{n} V}\right)^{2 / n} .
$$

Now we give an estimate of an upper bound for $c\left(r^{*}\right) \equiv C(k, n, \Omega)^{n / 2}$. By a "thick" set of $\mathbf{R}^{n}$ we mean a measurable set $E$ for which there exists a parallelepiped $\Pi$ with sides of length $a_{1}, a_{2}, \ldots, a_{n}$ parallel to coordinate axes and $\gamma>0$ such that

$$
|E \cap(\Pi+x)| \geq \gamma|\Pi|
$$

for every $x \in \mathbf{R}^{n}$. Let $J$ be another parallelepiped with sides of length $b_{1}, b_{2}, \ldots, b_{n}$ parallel to coordinate axes. If there exist $f \in L^{2}\left(\mathbf{R}^{n}\right)$, supp $\widehat{f} \subset J$, and a measurable set $E$ satisfying (2.3), then by Korvijkine [5, Theorem 3], we can assert that

$$
\|f\|_{L^{2}(E)} \geq\left(\frac{\gamma}{C^{n}}\right)^{C\left(n+\sum_{k=1}^{n} a_{k} b_{k}\right)}\|f\|_{L^{2}\left(\mathbf{R}^{n}\right)}
$$

holds for some constant $C=C(\gamma, n)>1$. Choose $E=\mathbf{R}^{n} \backslash B_{r^{*}}$ and $\Pi$ the cube with sidelength $2 r^{*}$ centered at 0 in $\mathbf{R}^{n}$. One can check that the formula (2.3) holds for $\gamma=1-\frac{\operatorname{Vol}\left(B_{r^{*}}\right)}{\operatorname{Vol}(\mathrm{II})}=1-\frac{\omega_{n}}{2^{n}}$. Since $\Omega$ is bounded, we can choose $J$ to be a 
cube of sidelength $D$ which covers $\Omega$. Since $\widehat{\phi_{j}} \in L^{2}\left(\mathbf{R}^{n}\right)$ and $\operatorname{supp} \widehat{\widehat{\phi}_{j}} \subset-\Omega \subset-J$, by the estimate (2.4),

$$
\left\|\widehat{\phi_{j}}\right\|_{L^{2}\left(\mathbf{R}^{n} \backslash B_{r^{*}}\right)}^{2} \geq\left(\frac{\gamma}{C^{n}}\right)^{2 C n\left(1+2 D r^{*}\right)}\left\|\widehat{\phi}_{j}\right\|_{L^{2}\left(\mathbf{R}^{n}\right)}^{2} .
$$

Equivalently,

$$
\int_{B_{r^{*}}}\left|\widehat{\phi_{j}}(z)\right|^{2} d z \leq(2 \pi)^{n}\left\{1-\left(\frac{\gamma}{C^{n}}\right)^{2 C n\left(1+2 D r^{*}\right)}\right\}\left\|\phi_{j}\right\|_{L^{2}\left(\mathbf{R}^{n}\right)}^{2} .
$$

Hence, $c\left(r^{*}\right)=(2 \pi)^{n}\left\{1-\left(\frac{\gamma}{C^{n}}\right)^{2 C n\left(1+2 D r^{*}\right)}\right\}<(2 \pi)^{n}$. This finishes the proof of Theorem 1.1.

\section{Proof of Theorem 1.2}

The proof of Theorem 1.2 is similar to that of Theorem 1.1. Let $\left\{\widetilde{\phi}_{j}\right\}_{j=1}^{k}$ be a set of orthonormal real-valued eigenfunctions corresponding to the eigenvalues $\widetilde{\mu_{1}}, \widetilde{\mu_{2}}, \ldots, \widetilde{\mu_{k}}$ and also consider the function defined by

$$
\widetilde{\Phi}_{k}(x, y) \equiv \sum_{j=1}^{k} \widetilde{\phi}_{j}(x) \widetilde{\phi}_{j}(y) \text {, for } x, y \in M^{m} .
$$

The projection of $\widetilde{h}_{z}(y) \equiv e^{i\langle z, y\rangle}\left(y \in M^{m} \subset \mathbf{R}^{n}, z \in \mathbf{R}^{n}\right)$ onto the subspace of $L^{2}(\Omega)$ spanned by $\widetilde{\phi}_{1}, \widetilde{\phi}_{2}, \ldots, \widetilde{\phi}_{k}$ can be written in terms of the Fourier transform $\widehat{\widetilde{\Phi}}_{k}$ of $\widetilde{\Phi}_{k}$ with respect to the $x$-variable $\left(x \in M^{m}\right)$ :

$$
\int_{M^{m}} e^{i\langle z, x\rangle} \widetilde{\Phi}_{k}(x, y) d x=\widehat{\widetilde{\Phi}}_{k}(z, y) .
$$

Hence following the same analysis in the proof of Theorem 1.1, one has

$$
\begin{aligned}
\widetilde{\mu}_{k+1} & \leq \inf _{r>0} \frac{\int_{B_{r}} \int_{M^{m}}\left|\nabla_{y}\left(\widetilde{h}_{z}(y)-\widehat{\widetilde{\Phi}_{k}}(z, y)\right)\right|^{2} d y d z}{\int_{B_{r}} \int_{M^{m}}\left|\widetilde{h}_{z}(y)-\widehat{\widetilde{\Phi}}_{k}(z, y)\right|^{2} d y d z} \\
& =\inf _{r>0} \frac{\int_{B_{r}} \int_{M^{m}}\left|\nabla_{y} h_{z}(y)\right|^{2} d y d z-\int_{B_{r}} \int_{M^{m}}\left|\nabla_{y} \widehat{\Phi_{k}}(z, y)\right|^{2} d y d z}{\int_{B_{r}} \int_{M^{m}}\left|h_{z}(y)-\widehat{\Phi}_{k}(z, y)\right|^{2} d y d z} \\
& \leq \frac{\frac{n \omega_{n} V}{n+2} r^{n+2}-\sum_{j=1}^{k} \widetilde{\mu}_{j} \int_{M^{m}}\left|\widetilde{\widetilde{\phi}}_{j}\right|^{2}}{\omega_{n} V r^{n}-\sum_{j=1}^{k} \int_{M^{m}}\left|\widetilde{\widetilde{\phi}}_{j}\right|^{2}}
\end{aligned}
$$

here we have exploited the fact that $\left|\nabla_{y} \widetilde{h}_{z}(y)\right| \leq|z|$ since $M^{m}$ is a regular submanifold of $\mathbf{R}^{n}$. By Theorem 7.1.27 in Hörmander [4, there exists a constant $C$ depending only on $M^{m}$ and the dimension $n$ such that for all $j \leq k$,

$$
\int_{B_{r}}\left|\widehat{\widetilde{\phi}}_{j}(z)\right|^{2} d z \leq C r^{n-m} \text {. }
$$

An estimate of an upper bound for the constant $C$ is also given later. Particularly, Plancherel's theorem asserts that $C=(2 \pi)^{n}$ if $m=n$. Set $\widetilde{r}=\left(\frac{k C}{\omega_{n} V}\right)^{1 / m}$. Let $r>\widetilde{r}$, from which we have

$$
\omega_{n} V r^{n}-k C r^{n-m}>0 .
$$


By Lemma 2.1,

$$
\frac{n \omega_{n} V}{n+2} r^{n+2}-C r^{n-m} \sum_{j=1}^{k} \widetilde{\mu}_{j} \geq 0,
$$

which yields

$$
\begin{aligned}
\sum_{j=1}^{k} \widetilde{\mu}_{j} & \leq \inf _{r>\widetilde{r}} \frac{n \omega_{n} V r^{m+2}}{(n+2) C} \\
& =\frac{n k}{n+2}\left(\frac{k C}{\omega_{n} V}\right)^{2 / m} .
\end{aligned}
$$

Finally, we show how to give an estimate of an upper bound for $C$. After using a partition we may assume that $M^{m}$ is covered by some finite open sets $\left\{U_{s}\right\}_{1 \leq s \leq q}$ such that in any $U_{s}$ the manifold $M^{m}$ is of the form

$$
\ddot{x}=h_{s}(\dot{x}) ; \dot{x}=\left(x_{1}, \ldots, x_{m}\right), \ddot{x}=\left(x_{m+1}, \ldots, x_{n}\right) .
$$

We may also assume there exist some finite smooth functions $\left\{\varphi_{s}\right\}_{1 \leq s \leq q}$ such that $\operatorname{supp} \varphi_{s} \subset U_{s}, 0 \leq \varphi_{s} \leq 1$ and $\sum_{s=1}^{q} \varphi_{s}=1$. Then $d S=a_{s}(\dot{x}) d \dot{x}$, where $a_{s}$ is some positive smooth function. A function $\phi$ defined on $U_{s}$ can be considered as a function of $\dot{x}$ with parameter $\ddot{x}$. Observe that

$$
\widehat{\phi}(z)=\int_{U_{s}} e^{i\left(\langle\dot{x}, \dot{z}\rangle+\left\langle h_{s}(\dot{x}), \ddot{z}\right\rangle\right)} \phi(\dot{x}, \ddot{x}) a_{s}(\dot{x}) d \dot{x} .
$$

For a fixed $\ddot{z}$ Parseval's formula gives

$$
\begin{aligned}
\int|\widehat{\phi}(z)|^{2} d \dot{z} & =(2 \pi)^{m} \int_{U_{s}}|\phi(\dot{x}, \ddot{x})|^{2} a_{s}(\dot{x}) d \dot{x} \\
& =(2 \pi)^{m} \int_{U_{s}}|\phi|^{2} a_{s}(\dot{x}) d S \\
& \leq(2 \pi)^{m} \sup _{U_{s}} a_{s} \int_{U_{s}}|\phi|^{2} d S .
\end{aligned}
$$

Integration with respect to $\ddot{z}$ for $|\ddot{z}| \leq r$ leads to

$$
\int_{B_{r}}|\widehat{\phi}(z)|^{2} d z \leq(2 \pi)^{m} \omega_{n-m} r^{n-m} \sup _{U_{s}} a_{s} \int_{U_{s}}|\phi|^{2} d S .
$$

Now let $\phi$ be a square integrable function defined on $M^{m}$. By (3.1), we have

$$
\begin{aligned}
\int_{B_{r}}|\widehat{\phi}(z)|^{2} d z & =\int_{B_{r}}\left|\sum_{s=1}^{q} \widehat{\left(\varphi_{s} \phi\right)}(z)\right|^{2} d z \\
& \leq\left. q \sum_{s=1}^{q} \int_{B_{r}} \widehat{\mid\left(\varphi_{s} \phi\right)}(z)\right|^{2} d z \\
& \leq q \sum_{s=1}^{q}(2 \pi)^{m} \omega_{n-m} r^{n-m} \sup _{U_{s}} a_{s} \int_{U_{s}}\left|\varphi_{s} \phi\right|^{2} d S \\
& \leq q(2 \pi)^{m} \omega_{n-m} r^{n-m} \sum_{s=1}^{q} \sup _{U_{s}} a_{s} \int_{M^{m}}|\phi|^{2} d S .
\end{aligned}
$$

Hence, $C=q(2 \pi)^{m} \omega_{n-m} \sum_{s=1}^{q} \sup _{U_{s}} a_{s}$. This concludes the proof of Theorem 1.2. 


\section{ACKNOWLEDGMENT}

The first author is greatly indebted to Professor Fuquan Fang, whose guidance and support were crucial for the successful completion of this paper.

\section{REFERENCES}

[1] E. B. Davies, Heat Kernels and Spectral Theory. Cambridge Univ. Press, Cambridge, 1989. MR.1103113 (92a:35035)

[2] V. P. Havin, B. Jöricke, The Uncertainty Principle in Harmonic Analysis, Springer-Verlag, Berlin, 1994. MR1129019 (93e:42001)

[3] L. Hermi, Research Statement - December 2003. http://math.arizona.edu/ hermi/rp.pdf.

[4] L. Hörmander, The Analysis of Linear Partial Differential Operators. I. Distribution Theory and Fourier Analysis, Springer-Verlag, Berlin, 1983. MR0717035 (85g:35002a)

[5] O. Kovrijkine, Some results related to the Logvinenko-Sereda theorem, Proc. Amer. Math. Soc. 129(2001), 3037-3047. MR1840110 (2003c:46031)

[6] P. Kröger, Upper bounds for the Neumann eigenvalues on a bounded domain in Euclidean space, J. Funct. Anal. 106(1992), 353-357. MR1165859 (93d:47091)

[7] P. Li, S. T. Yau, On the Schrödinger equation and the eigenvalue problem, Comm. Math. Phys. 88(1983), 309-318. MR0701919 (84k:58225)

[8] A. Melas, A lower bound for sums of eigenvalues of the Laplacian, Proc. Amer. Math. Soc. 131(2003), 631-636. MR1933356(2003i:35218)

[9] R. Schoen, S. T. Yau, Lectures on Differential Geometry, International Press, Boston, 1994. MR.1333601 (97d:53001)

[10] H. Weyl, Das asymptotische Verteilungsgesetz der Eigenwerte linearer partieller Differentialgleichungen. Math. Ann. 71(1912), 441-469. MR.1511670

Nankai Institute of Mathematics, Nankai University, Tianjin 300071, People's RePUBLIC OF CHINA

E-mail address: liliangpan@yahoo.com.cn

Department of Mathematics, Xidian University, Xi'an 710071, People's Republic of CHINA

Current address: Department of Mathematics, University of Texas at Austin, Austin, Texas 78712

E-mail address: ltang@math.utexas.edu 